\title{
Attention Enhancement and Motion Assistance for Virtual Reality-Mediated Upper-limb Re- habilitation
}

\author{
Min Li, Wenliang Guo, Jian Xu, Shuai Xue, Di Wu, Ziting Liang, Hua Yuan, Jun Xie, Guanghua Xu, \\ Kaspar Althoefer
}

\begin{abstract}
Dysfunctions of upper limbs caused by diseases such as stroke result in difficulties in conducting day-to-day activities. Studies show that rehabilitation training using virtual reality games is helpful for patients to restore arm functions. It has been found that ensuring active patient participation and effort devoting in the process is very important to obtain better training results. This paper introduces a method to help patients increase their engagement and provide motion assistance in virtual reality-mediated upper-limb rehabilitation training. Attention enhancement and motion assistance is achieved through an illusion of virtual forces created by altering the drag speed between the cursor and the object presented on a screen to the patient as the only feedback. We present two game forms using the proposed method, including a target-approaching game and a maze-following game. The results of evaluation experiments with human participants showed that the proposed method could provide path guidance that significantly improved path-following performance of users and required more involvement of the users when compared to playing the game without attention enhancement and motion assistance.
\end{abstract}

Index Terms-Upper-limb rehabilitation, virtual reality-mediated rehabilitation, attention enhancement.

\section{INTRODUCTION}

$\mathrm{D}$ ISEASES such as cerebral palsy, stroke, loss of proprioception, post tendon surgery, spinal cord injury, and multiple sclerosis may cause dysfunction of upper limbs, and thus, result in difficulties in carrying out activities of daily living (ADLs) [1]. Upper-limb rehabilitation aims to help patients regain motor functions and empower them to live independently [2]. Virtual reality (VR) allows patients to interact with simulated environments and perceive real-time performance feedback during rehabilitation [3]. VR-mediated motor interventions have been shown to be effective in motor rehabilitation [3]. Commercialized VR gaming devices such as the Kinect sensor (Microsoft Corporation, Albuquerque, New Mexico) have been used in self-training and evaluation systems for motion rehabilitation [4], [5]. With such self-training and evaluation systems available, motor rehabilitation training at home is becoming a real possibility allowing patients to con-

This work was supported by the National Natural Science Foundation of China (51975451). M. Li, W. Guo, J. Xu, S. Xue, D. Wu, Z. Liang, G. Xu, and J. Xie are with School of Mechanical Engineering, Xi'an Jiaotong University, Shaanxi, 710049 China (e-mail: min.li@mail.xjtu.edu.cn). H. Yuan is with Department of Rehabilitation, Xijing Hospital, Xi'an 710032, China. K. Althoefer is with Faculty of Science \& Engineering, Queen Mary University of London, London E1 4NS, UK. tinue with their rehabilitation beyond the hospital [4], [5].

Rehabilitation professionals generally believe that it is very important to ensure that patients actively participate and put more effort into the exercise training process to induce neuroplasticity and thus promote motor rehabilitation [6]. More attention and involvement of the patients during rehabilitation training are desired to achieve better outcomes [7]. However, the commonly used repetitive movement rehabilitation exercises are often boring. How to increase patient engagement in rehabilitation training needs more investigation. We argue that the training process needs to be more interactive and participatory to enhance the patient's attention during the process, to make the patient put more effort into the exercise.

Patients with weakened arms would benefit from confidence building through performance improvement achieved via motion assistance or path guidance during VR-mediated rehabilitation training, since the brain reward system is implicated during motor learning and neuroplasticity [8]. To provide motion assistance or path guidance for rehabilitation, expensive robotic devices or haptic feedback devices are normally required [2]. How to provide low-cost solutions of motion assistance for VR-mediated at-home motor rehabilitation is being investigated here.

Most current VR systems built for rehabilitation provide primarily visual experiences. Through an interactive process, synchronously integrating visual feedback with the actions of the user employing pseudo-haptic feedback can create virtual forces without using physical haptic devices [9], [10]. Bringing a visual-haptic illusion to VR-mediated motor rehabilitation may enrich the patient experience, strengthen the stimulation, increase patient engagement, and provide virtual motion assistance/resistance to the arm movements.

In this paper, we propose a method to add motion assistance to the arm movements through visual-haptic illusion during the training process of VR-mediated upper-limb rehabilitation. By providing virtual resistance to the avatar motion in the rehabilitation game, the user's performance is expected to be improved and thus the user is more confident to actively participate in the training. The effects of virtual motion assistance on the game participants during the training process is examined by comparing hand trajectories with and without virtual motion assistance. The attention level of the participants is introduced as an evaluation indicator to investigate the attention enhancement effect of the proposed method on the participants.

Permission from IEEE must be obtained for all other users, including reprinting / republishing this material for advertising or promotional purposes, creating new

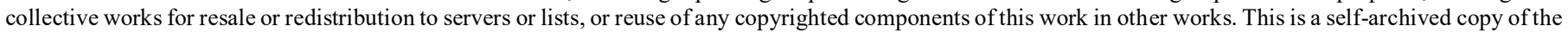
submitted version of the above paper. There are minor differences between this and the published version. 
https://ieeexplore.ieee.org/document/9223704

\section{MATERIALS AND MethodS}

A. VR-mediated motor rehabilitation training with attention enhancement and motion assistance

Controlling arms to drive hands towards certain targets and following certain paths are very important upper-limb functions when conducting day-to-day activities. The process of practicing those movements is associated with learned, biologically valid actions rather than fragmented movements commonly used in current rehabilitation, and thus can promote the reorganization and recovery of motor function after stroke [11]. In this study, we propose a target-approaching game and a maze-following game employing visual-haptic illusion using pseudo-haptic feedback for upper-limb rehabilitation. In the target-approaching game (see Fig.1 (a)), the game player uses a cross-shaped cursor to drag a small square object starting from the bottom line of the game window on a computer screen to touch several targets appearing in higher screen positions one after another. During the process, the ratio of the cursor-movement speed to the hand-movement speed is constant while the ratio of the object-movement speed to hand-movement speed changes according to our pseudo-haptics algorithm. If the object follows the cursor tightly, it will cause the feeling in the participant that the object is easy to drag. If the object moves slower than the cursor, it will cause the feeling that the object is hard to drag, and thus, virtual resistance is experienced. The virtual resistance employed by the proposed method increases as the offset between the cursor and the object increases when the patient drags the object away from the bottom line of the game window towards the targets. When the object reaches the highest target, the object stops following the cursor towards higher positions.

The maze-following game is designed for patients to practice their path-following skills. In the maze-following game, the game player uses a cross-shaped cursor to drag a small square object following the centerline of the maze path from the start point to the end of the maze to complete the maze-following game (see Fig. 1 (b)). Because of conditions like arm weakness, poor proprioception or hand trembling, it is difficult for patients to keep the object close to the centerline of the path at all times. In the maze-following game, the object speed decreases as the distance between the object and the center of the path increases. Therefore, virtual resistance to the arm is experienced when the arm moves away from the centerline of the path. In other words, virtual path guidance is provided. The object stops following the cursor towards the outside of the path when it touches the edges of the path.

A DELUX M168 vertical computer mouse (Delux Technology Co., Ltd, Shenzhen, China) is used here as the input device to mimic the feeling of holding a handle that is commonly used in upper-limb rehabilitation devices [12], [13]. The positioning accuracy of this mouse is $1600 \mathrm{dpi}$. The relationship between the moving distance of the cursor on the screen and the moving distance of the hand was 62.99 pixel $/ \mathrm{mm}$. However, a computer mouse can only capture the user's hand movement on a horizontal plane. Therefore, the rehabilitation training motion using a computer mouse can be only conducted on a horizontal plane. To lift this restriction, other motion capturing devices are required. The Kinect sensor 2.0, a low-cost motion capture device, has been used in VR-mediated rehabilitation [4], [5]. The Kinect sensor 2.0 tracks 25 joints of the human body with three-dimensional coordinates. Here, only the coordinates of the user's left or right hand are used. After the coordinates of the user's left or right hand are acquired, the relevant Windows API is called to activate the mouse cursor movements on the screen. The range of motion of the arm in the game should reach a certain size for the purpose of rehabilitation training but not too large for the patient to accomplish or cause any harm. In this study, the horizontal coordinates of the game window are mapped to 3.5 times the length from neck to head. The vertical coordinates of the game window are mapped to twice the length from neck to head. Thus, the spatial coordinates of the hand are mapped to the screen coordinates of the cursor. To control the cursor to finish the maze requires the user to move the hand a distance that is more than 4 times the length from his/her neck to head. These ratios can be adjusted in the program according to the different states of the patient.
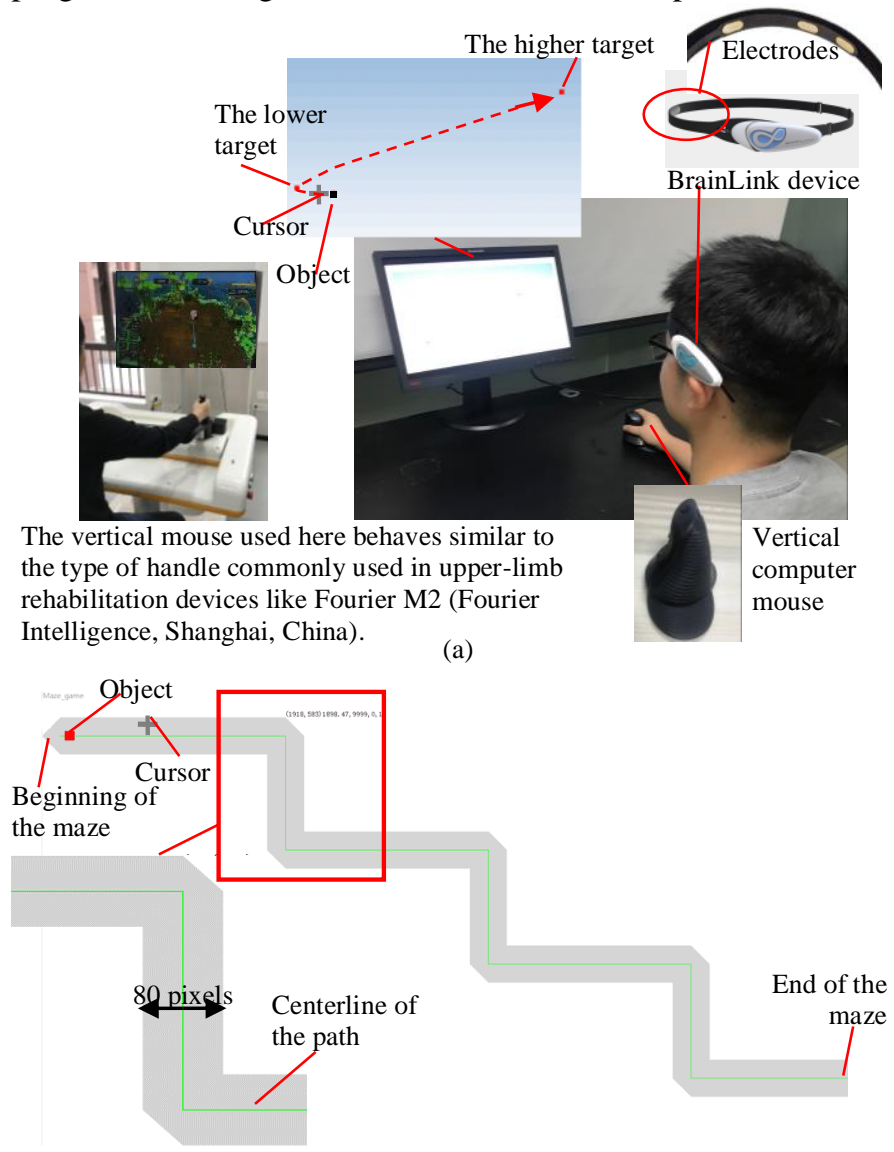

(b)

Fig. 1 The interface of (a) target-approaching game and (b) maze-following game for upper-limb rehabilitation.

\section{B. Experiments}

Three evaluation experiments were conducted to prove the feasibility of the proposed method enhancing patient attention for VR-mediated upper-limb rehabilitation. Twenty healthy adults were recruited as subjects for our study. Participants range in age from 20 to 25 . The dominant hand of each par- 
https://ieeexplore.ieee.org/document/9223704

ticipant is the right hand. Before the experiment, participants knew nothing about pseudo-haptic feedback. This study was approved by the institutional review board of Xi'an Jiaotong University. Participants signed a written consent form before conducting the experiments that were part of this study. Before the experiment, participants were asked to conduct some physical exercises like push-ups to cause muscle fatigue to their upper limbs to simulate the conditions of muscle weakness.

During the first two experiments, participants were asked to sit in front of a desktop computer and hold the vertical mouse in their palm. They moved the vertical mouse across a tabletop, aiming to control the cursor to drag the small square object to touch targets located in higher positions on the game window (in the target-approaching game) or to follow the path in a given maze keeping as close as possible to the centerline of the path (in the maze-following game). In the target-approaching game, the ratio of the object speed to the cursor speed was mapped to the distance between the object and the bottom line of the game window. In the maze-following game, the ratio of the object speed to the cursor speed was mapped to the distance between the object and the maze path center. In the experiments, four mapping relations conducted in a pseudo-random order, including linear relation, polynomial relation, Gaussian relation, and rational interpolation, were evaluated to find out the most effective one for the specific participant. Participants were asked with which mapping relation they could feel the most powerful virtual force. After that experiments were conducted to evaluate the performances of the participants in the target-approaching game and the maze-following game with and without the proposed pseudo-haptic feedback; when the pseudo-haptic effect was active, it was based on each participant's selected and most effective mapping relation. Each participant tested each mode three times. The participant's attention was recorded using a Brainlink Lite device (Macrotellect Ltd., Shenzhen, China). The attention levels are presented as a unitless parameter, ranging from 0 (distracted) to 100 (focused). The attention levels of each participant were recorded so that we could analyze whether the participant was focused on the rehabilitation process or not. During the maze-following experiment, the trajectories of the cursor and the small square object were recorded. Trajectory offsets were calculated as the distance between the centerline of the maze path and the trajectory of the cursor and the object.

Another experiment was conducted to test the feasibility of using the Kinect sensor to capture the hand position of the participant for VR-mediated rehabilitation with attention enhancement and motion assistance. Participants were asked to stand in front of a desktop computer with the same computer monitor as used in the other experiments. The Kinect sensor was placed in front of the participant at a distance of 1.5 meters (see Fig. 2). In the beginning of the experiment, participants had a chance to get familiar with the cursor control using the Kinect sensor until they feel confident with the system and its control. Then the same target-approaching game as used in the experiments with the vertical mouse as the input device was employed here. They were asked whether they could feel the virtual force when they drag the object towards the two targets.
Then, the same four mapping relations as in the experiment set 1 were evaluated to find out the most effective one for the specific participant using the Kinect. Participants were asked which mapping caused the strongest virtual force.

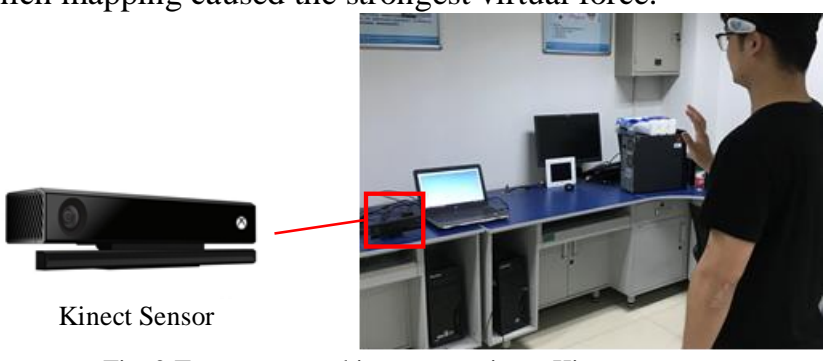

Fig. 2 Target approaching game using a Kinect sensor

\section{RESULTS}

In the target-approaching game, seventeen subjects $(85 \%)$ claimed that they could feel a stronger virtual force with the rational interpolant relation. Two participants $(10 \%)$ chose the Gaussian interpolant relation to be the most effective mapping relation. One subject considered the linear interpolant as the most useful one. All the participants had the same choice of mapping relation in the maze-following game as in the target-approaching game.

The attention levels of the participants collected by the Brainlink device during the experiment are shown in Fig. 3 and Table I. The average attention level in those two games with attention enhancement and motion assistance was significantly higher than that in the games without attention enhancement and motion assistance. This result shows that the introduction of attention enhancement and motion assistance to the target-approaching game and the maze-following game required more attention and involvement of the participants than when playing the game without using attention enhancement and motion assistance.

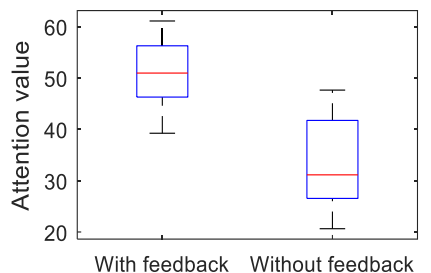

(a)

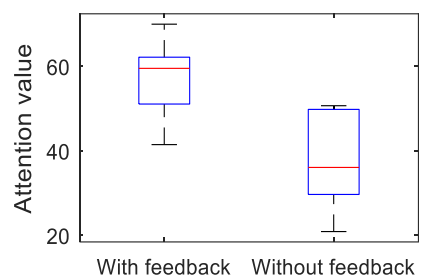

(b)
Fig. 3. The attention levels of the participants in the (a) target-approaching game and (b) maze following game.

TABLE I

StatisticAl RESUlts of THE AVERAGE ElAPSED Time AND ATtENTION LEVELS DURING THE GAMES

\begin{tabular}{llll}
\hline \hline & $\begin{array}{l}\text { With the pro- } \\
\text { posed attention } \\
\text { enhancement } \\
\text { method }\end{array}$ & $\begin{array}{l}\text { Without the } \\
\text { proposed atten- } \\
\text { tion enhance- } \\
\text { ment method }\end{array}$ & $\begin{array}{l}\text { Pairwise } \\
\text { student t-test } \\
\text { p-value }\end{array}$ \\
\hline $\begin{array}{l}\text { Average attention levels in } \\
\text { target-approaching game }\end{array}$ & $51.16 \pm 7.24$ & $33.01 \pm 8.43$ & $6.46 \times 10^{-9 * * *}$ \\
$\begin{array}{l}\text { Average attention levels in } \\
\text { maze following game }\end{array}$ & $56.63 \pm 8.0$ & $36.79 \pm 9.72$ & $2.85 \times 10^{-8 * *}$ \\
\hline
\end{tabular}

maze following game

Stronger significance than the $1 \%$ level

Fig. 4 shows the average cursor trajectory and the object trajectory compared to the centerline of the maze path in the maze-following game. The trajectory offset without the pro-

Permission from IEEE must be obtained for all other users, including reprinting / republishing this material for advertising or promotional purposes, creating new

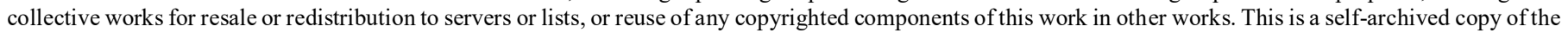
submitted version of the above paper. There are minor differences between this and the published version. 
https://ieeexplore.ieee.org/document/9223704

posed attention enhancement and motion assistance method was much larger (average 13.05 \pm 2.06 ) than that in the mode with attention enhancement and motion assistance (the cursor average trajectory offset $5.65 \pm 1.44$ and the object average trajectory offset $4.04 \pm 0.92$ ). A pairwise student t-test with Bonferroni correction [14] was performed to test the significance of the difference among those three trajectory offsets. With Bonferroni correction, the significance level was $0.05 / 3=0.017$. The results are shown in Table II. Strong significances of the differences were found. The results indicate that the maze-following game with the proposed attention enhancement and motion assistance method reduces the trembling of the participants' hands caused by muscle fatigue because of the push-ups before the experiment and improves their path-following performance when compared to the mode without attention enhancement and motion assistance.

In the Kinect sensor experiment, all twenty subjects could use the Kinect sensor to control the cursor after a short time of practice although they claimed that moving the cursor with the Kinect sensor was more difficult than using a virtual mouse. Twenty subjects claimed that it was obvious to feel the virtual force as they moved the cursor to drag the object towards the two targets in the target-approaching game. Sixteen subjects $(80 \%)$ chose the rational interpolant mapping relation as the most effective one, three $(15 \%)$ chose the Gaussian interpolant mapping relation and one $(5 \%)$ considered that the linear interpolation mapping relation to be the best.

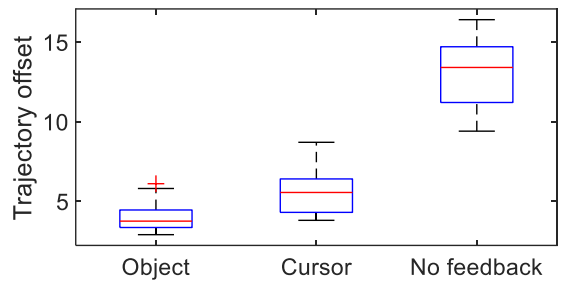

Fig. 4. The statistical result of the cursor trajectory offset and the object trajectory offset compared to the centerline of the maze path.

TABLE II

STUDENT T-TEST FOR THE TRAJECTORY OFFSET TO THE CENTERLINE OF THE MAZE PATH

\begin{tabular}{lc}
\hline \multicolumn{1}{c}{ Item } & p-value \\
\hline \hline $\begin{array}{l}\text { Cursor trajectory offset with attention enhancement } v s . \\
\text { without attention enhancement }\end{array}$ & $1.55 \times 10^{-14 * *}$ \\
$\begin{array}{l}\text { Object trajectory offset with attention enhancement } v s . \\
\text { without attention enhancement }\end{array}$ & $6.27 \times 10^{-14 * *}$ \\
$\begin{array}{l}\text { Cursor trajectory offset } v s . \text { Object trajectory offset with } \\
\text { attention enhancement }\end{array}$ & $8.99 \times 10^{-7 * * *}$ \\
\hline ** Stronger significance than the 1\% level
\end{tabular}

\section{CONCLUSIONS}

A low-cost solution to provide attention enhancement and motion assistance for VR-mediated upper-limb rehabilitation is proposed in this paper. The evaluation experiments showed that the introduction of attention enhancement and motion assistance to upper-limb rehabilitation games required more attention and involvement of the users when compared to playing the game without attention enhancement and motion assistance; the introduction of the proposed attention enhancement and

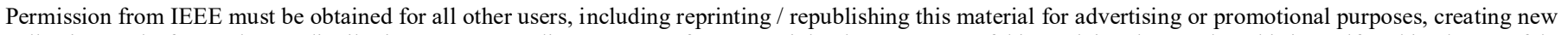

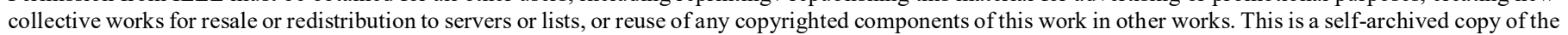
submitted version of the above paper. There are minor differences between this and the published version. motion assistance method in the maze-following game improved the participants' path-following performance when compared to the mode without attention enhancement and motion assistance; most participants claimed that a stronger virtual force could be felt when using the rational interpolant speed mapping relation in the pseudo-haptic feedback compared to when using the linear relation, the polynomial relation or the Gaussian relation.

Further studies will be conducted to further investigate the upper-limb trembling reducing mechanism of the proposed attention enhancement and motion assistance method. More targets in the target-approaching game and more complex maze maps involving rightward, leftward, upward, and downward motions in the maze-following game will be used in further studies. Real patients will be involved in future studies. Rational interpolant speed mapping curves with different degrees of steepness will also be studied in more detail.

[1] T. Higuma, K. Kiguchi, and J. Arata, "Low-Profile Two-Degree-of-Freedom Wrist Exoskeleton Device Using Multiple Spring Blades," IEEE Robot. Autom. Lett., vol. 3, no. 1, pp. 305-311, 2018.

[2] M. Li, G. Xu, J. Xie, and C. Chen, “A review: Motor rehabilitation after stroke with control based on human intent," Proc. Inst. Mech. Eng. Part H J. Eng. Med., vol. 232, no. 4, pp. 344-360, 2018.

[3] S. Cho et al., "Development of virtual reality proprioceptive rehabilitation system for stroke patients," Comput. Methods Programs Biomed., vol. 113, no. 1, pp. 258-265, 2014.

[4] W. Pei, G. Xu, M. Li, H. Ding, S. Zhang, and A. Luo, "A motion rehabilitation self-training and evaluation system using Kinect," in 13th International Conference on Ubiquitous Robots and Ambient Intelligence (URAl), 2016, pp. 353-357.

[5] D. S. Park, D. G. Lee, K. Lee, and G. C. Lee, "Effects of Virtual Reality Training using Xbox Kinect on Motor Function in Stroke Survivors: A Preliminary Study," J. Stroke Cerebrovasc. Dis., 2017.

[6] K. K. Ang and C. Guan, "Brain-Computer Interface in Stroke Rehabilitation,” J. Comput. Sci. Eng., vol. 7, no. 2, pp. 139-146, 2013.

[7] A. Basteris, N. S. M, A. H. Stienen, J. H. Buurke, P. G. B, and A. Farshid, "Training modalities in robot-mediated upper limb rehabilitation in stroke: a framework for classification based on a systematic review," $J$. Neuroeng. Rehabil., vol. 11, no. 1, p. 111, 2014.

[8] L. Piggott, S. Wagner, and M. Ziat, "Haptic Neurorehabilitation and Virtual Reality for Upper Limb Paralysis: A Review," Crit Rev Biomed Eng., vol. 1-2, no. 44, pp. 1-32, 2016.

[9] D. A. G. Jauregui, F. Argelaguet, A.-H. Olivier, M. Marchal, F. Multon, and A. Lecuyer, "Toward 'pseudo-haptic avatars': modifying the visual animation of self-avatar can simulate the perception of weight lifting.," IEEE Trans. Vis. Comput. Graph., vol. 20, no. 4, pp. 654-661, Apr. 2014.

[10] A. Lécuyer, J.-M. Burkhardt, and C.-H. Tan, "A study of the modification of the speed and size of the cursor for simulating pseudo-haptic bumps and holes," ACM Trans. Appl. Percept., vol. 5, no. 3, pp. 1-21, Aug. 2008.

[11] G. Buccino, A. Solodkin, and S. L. Small, "Functions of the mirror neuron system: implications for neurorehabilitation.," Cogn. Behav. Neurol., vol. 19, no. 1, pp. 55-63, 2006.

[12] Fourier Intelligence, “ArmMotus ${ }^{\mathrm{TM}} \mathrm{M} 2, " 2019$. [Online]. Available: http://www.fftai.com/product_en/M2.php. [Accessed: 20-Dec-2019].

[13] P. S. Lum, C. G. Burgar, P. C. Shor, M. Majmundar, and M. Van der Loos, "Robot-assisted movement training compared with conventional therapy techniques for the rehabilitation of upper-limb motor function after stroke," Arch. Phys. Med. Rehabil., 2002.

[14] R. G. J. Miller, Simultaneous Statistical Inference. New York: Springer-Verlag, 1991.

\section{REFERENCES}

